\title{
写好部断性meta分析的秘密 (一)
}

徐莉

选择去学习诊断性 meta 是我人生里最正确的选择之一! 2013 年 12 月我开始学习, 到 2014 年 1 月份发表了我的第一篇诊断性 meta 分析, 3 分的杂志, 4 天直接接受, 然后便一直在这条路上走下去了。时至今日, 两年多的时间, 有些东西已经跑进我的脑子里挥之不去, 很多很多, 在 我听到一首好听的音乐的时候，在我看见很美的照片的时候，在我弹钢 琴的时候，在我跳舞的时候，我会时不时的想起诊断性 meta，因为美 的东西都是共通的。

怎么样去写成一篇好的诊断性 meta，现在的我只能感觉到它们， 却不能细化地说出来, 但它们在发酵, 我需要等待美酒酿成, 把这些 美的东西串起来，然后发现。如果一篇文章就是一个故事，那么我会用 我独特的眼光和理解来给你讲, 下面的图片将结合实际, 深入浅出地 分享诊断性 meta 分析……希望大家都能尽快上手。

\section{这个主题别人写过怎么办? 一一剪裁和聚焦}
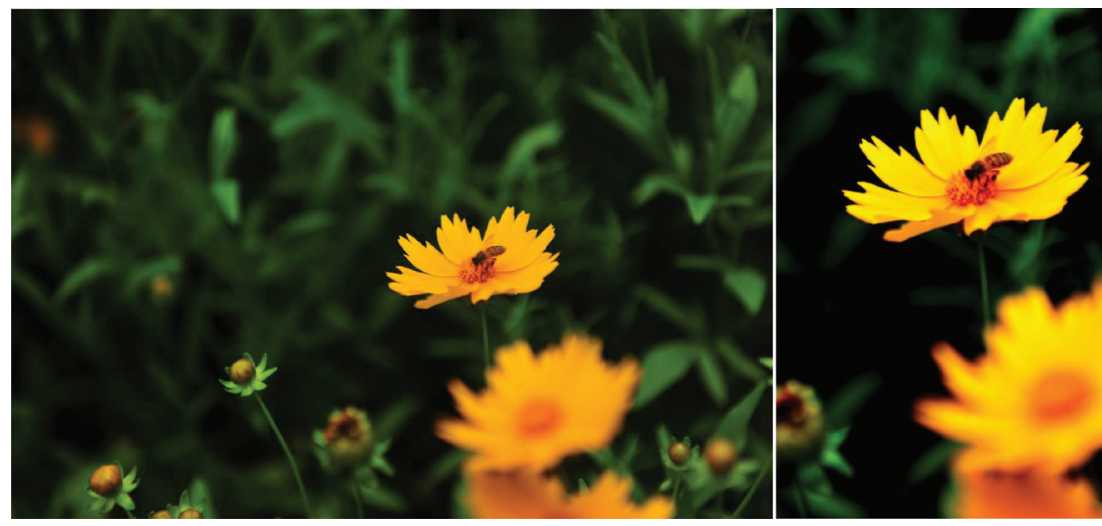

简单动作怎么做?

在影像学中, 当磁共振波谱 (MRS, 测定活体内某一特定组织区 域代谢物含量的无创性技术) 和弥散加权成像 (DWI, 利用水分子的弥 散运动特性进行成像) 刚兴起的时候, 随便应用于一个器官, 就是很 好的文章。现在又发展到体素内不相干运动成像 (IVIM, 无创评价活 体组织内分子扩散运动及灌注的 MRI 新技术 ) 定量动态增强热门的时 候。我们以此来作为素材了解下什么是简单动作。

所谓简单的动作，就是针对单个的技术 ( 如磁共振波谱，弥散加
权成像，可以替换为任意的其他 的诊断技术）而言，提取四格表 数据, 获得真阳性 (true positive, TP), 真 阴 性 (false positive, FP)，假阳性、假阴性数据（false negative cases, FN), 然后通过 软件得出数据。这个简单动作, 其实是比较简单、容易、无多大 技术含量的。

常用的软件: Meta-DiSc software and Stata

推荐用书: 《meta 分析软件应 用与实例解析》

当对于简单的动作了解和掌 握后, 又会随之而来如下问题:

1. 难度动作怎么做?

2. 简单的合成已经会了, 我们又 应该怎么对待呢?

3. 学完就扔, 还是反复练习?

4. 反复练习后又应该怎样去提 高呢?

简单的动作在开始的时候, 个人感觉挺好发文章的, 到了后 来就感觉越来越难了, 因为好吃 的肉都被大家吃了, 慢慢的需要 加入越来越多的变化, 越来越细 化, 才能吸引审稿人通过审稿。

而难度动作 $=$ 简单动作的复 合

\section{Practice makes perfect!}

笔者将在下一次开始谈这个 问题，欢迎大家继续关注本刊。 